\title{
Day 14 Intervention for Penetrating Brain Injury with a Good Glasgow Coma Scale Score: A Case Report
}

\author{
Mai Matsubara ${ }^{1}$, Yoshiaki Sakamoto ${ }^{1}$, Satoshi Takahashi ${ }^{1}$, Yu Ota $^{1}$, and Kazuo Kishi ${ }^{1}$ \\ ${ }^{1}$ Keio University School of Medicine Graduate School of Medicine
}

August 21, 2021

\begin{abstract}
Penetrating brain injury is a rare pathology generally requiring emergency surgical intervention. We discuss a case of penetrating brain injury by the umbrella in which surgical intervention was performed 14 days after the injury, and obtained good clinical results.
\end{abstract}

\section{Day 14 Intervention for Penetrating Brain Injury with a Good Glasgow Coma Scale Score: A} Case Report

Mai Matsubara, M.D. ${ }^{1}$, Yoshiaki Sakamoto, M.D. ${ }^{1}$, Satoshi Takahashi, M.D. ${ }^{2}$, Yu Ota, M.D. ${ }^{3}$ Kazuo Kishi, M.D. ${ }^{2}$

${ }^{1}$ Department of Plastic and Reconstructive Surgery, Keio University School of Medicine, 35 Shinanomachi, Shinjuku-ward, Tokyo 160-8582, Japan

${ }^{2}$ Department of Neurosurgery, Keio University School of Medicine, 35 Shinanomachi, Shinjuku-ward, Tokyo 160-8582, Japan

${ }^{2}$ Department of Opthalmology, Keio University School of Medicine, 35 Shinanomachi, Shinjuku-ward, Tokyo 160-8582, Japan

\section{Corresponding author}

Yoshiaki Sakamoto, M.D.

Department of Plastic and Reconstructive Surgery

Keio University School of Medicine

35 Shinanomachi, Shinjuku-ward, Tokyo 160-8582, Japan

Tel: +81-3-5363-3814 Fax: +81-3-3352-1054

E-mail: ysakamoto@z8.keio.jp

\begin{abstract}
Penetrating brain injury is a rare pathology generally requiring emergency surgical intervention. We discuss a case of penetrating brain injury by the umbrella in which surgical intervention was performed 14 days after the injury, and obtained good clinical results.
\end{abstract}

\section{Key Clinical Message}


Emergency surgical intervention is usually required for brain injuries. However, in this case, surgical intervention was performed 14 days after injury occurrence. If the Glasgow Coma Scale is high, as in this case, emergency surgery is not necessary. Instead, selective surgery or conservative management should be sufficient.

Keywords: penetrating brain injury, non-missile injury, surgical intervention, superior orbital fracture, guidelines

\section{INTRODUCTION}

Penetrating brain injuries include traumatic brain injuries, except those caused by blunt-force mechanisms ${ }^{1}$. This can be divided into missile and non-missile injuries. Missile injuries are caused by high-velocity objects ${ }^{2}$, while non-missile injuries result from violence, accidents, or suicidal attempts with low-velocity objects ${ }^{3}$. Penetrating brain injury has a poor prognosis. Therefore, the guidelines for managing this form of injury have been standardized ${ }^{4}$.

Neurosurgeons and emergency physicians perform the management of penetrating brain injury. However, craniofacial surgeons are not typically involved. To the best of our knowledge, no previous studies have described penetrating brain injury management from a craniofacial surgeon's perspective. We discuss a case of non-missile penetrating brain injury and the optimal time for surgical intervention.

\section{CASE PRESENTATION}

A 53-year-old male patient with right orbital prolapse, right oculomotor nerve palsy, and right upper eyelid laceration due to a stab injury by an umbrella tip was admitted to our hospital. Disturbance of consciousness was not observed, and the patient had a Glasgow Coma Scale (GCS) score of 15.

Immediate cranial computed tomography revealed right retrobulbar and frontal subarachnoid hemorrhage, right superior orbital wall fracture, and right frontal lobe contusion (Fig. 1, 2). A neuro-ophthalmologic evaluation revealed loss of vision in the right eye.

The umbrella tip was removed immediately after the injury by a third person, and the patient was fully conscious. Broad-spectrum antibiotic therapy was initiated without performing early surgical intervention.

However, cerebrospinal fluid leakage was observed, and the eyeball edema decreased. Hence, surgery was scheduled on day 14 .

The operation was performed by a multidisciplinary team consisting of neurosurgeons, ophthalmologists, and craniofacial surgeons. Following right orbit enucleation, debridement and irrigation were performed at the site of the penetrating wound. Subsequently, the $2 \times 1.5-\mathrm{cm}$ skull base defect was reconstructed with a split cranial bone graft. Two different pericranial flaps were used to patch the dural opening and shield the paranasal sinus.

After wound closure, a dressing was applied. No complications were observed during the postoperative period. However, mental counseling was required. The patient was discharged from the hospital 58 days after injury.

After 6 months, the patient underwent further surgery for aesthetic eyelid revision. The patient was able to resume his daily activities (Fig. 1,2).

\section{DISCUSSION}

Penetrating brain injury is a rare pathology. Compared to missile injury, non-missile injury has a more favorable outcome since the primary lesion is more localized ${ }^{3}$.

Objects that can cause non-missile injuries include knives, pitchforks, crochet hooks, knitting needles, breech pins, umbrellas, crowbars, iron rods, toilet brush handles, chopsticks, flatware, screwdrivers, keys, car antenna aerials, and scissors ${ }^{5}$. These objects have insufficient kinetic energy and velocity to penetrate the cranium ${ }^{1,5}$. Therefore, most reported cases of non-missile injuries involve injuries that have trajectories through the orbit $^{6}$. 
Immediate (preferably within 12 hours) life-saving surgery has been recommended for penetrating brain injury patients ${ }^{1}$. However, there are no consistent evidence-based criteria for the type or timing of surgical intervention. Furthermore, a previous study reported that surgical intervention did not significantly change the survival rate. Various times for surgical intervention $(<12$ hours to 11 days $)$ were recommended for penetrating brain injury patients with high GCS scores ${ }^{7}$.

In contrast, early surgical intervention is not recommended for patients with low GCS because a significant relationship between operative intervention and survival was found in patients with low GCS scores on $\operatorname{admission}^{7}$.

In the present case, surgical intervention was performed 14 days after the injury. This was later than the optimal time, according to the penetrating brain injury guidelines. Since the patient had a high GCS score on admission, there was a possibility that the prognosis would improve without surgical intervention. The patient exhibited traumatic cerebrospinal fluid leakage, which typically improves with conservative therapy. Hence, conservative antibiotic therapy was initiated. Meningitis did not occur, but cerebrospinal fluid leakage persisted. Therefore, surgery was performed on day 14 .

The operator's assessment of salvageability largely influences the decision-making for penetrating brain injury treatment ${ }^{8}$. Penetrating brain injury management remains poorly described since it is based on two-decadeold guidelines ${ }^{3,4}$. Since penetrating brain injury is related to survival, conducting a randomized control study was challenging, and only low-quality literature, such as retrospective studies and case series, are available. However, these studies can still be used to create new guidelines.

\section{CONCLUSION}

In conclusion, we discussed a case of a non-missile penetrating brain injury treated surgically 14 days after the injury. To our knowledge, this was the most recent report on a surgical intervention with good results. Hence, new guidelines may be necessary. These guidelines should be based on expert opinion and sufficient evidence.

\section{DECLARATIONS}

Ethics approval and consent to participate: N/A

Consent for publication: Written informed consent was obtained from the patient for publication of this case report and any accompanying images. A copy of the written consent is available for review by the Editor-in-Chief of this journal.

Availability of data and materials: N/A

Competing interests: None

Funding: None

Acknowledgments: None

REFERENCES

1. Esposito, D. P., and J.P. Walker. 2009. Contemporary management of penetrating brain injury. Neurosurgery Quarterly 19:249-254.

2. Gutierrez-Gonzalez, R., G. R. Boto, M. Rivero-Garvia, A. Perez-Zamarron, and A. Gomez. 2008. Penetrating brain injury by drill bit. Clinical Neurology and Neurosurgery 110:207-210.

3. 2001. Part 2. Prognosis in penetrating brain injury. Journal of Trauma 51(2 Suppl):S44-S86.

4. 2001. Part 1. Guidelines for the management of penetrating brain injury. Introduction and methodology. Journal of Trauma 51(2 Suppl):S3-6.

5. Sweeney J.M., J. J. Lebovitz, J. L. Eller, J. R. Coppens, R. D. Bucholz, and S. I. Abdulrauf. 2011. Management of non-missile penetrating brain injuries: A description of three cases and review of the literature. Skull Base Reports 1:39-46. 
6. Turbin R. E., D. N. Maxwell, P. D. Langer, B. Hubbi, L. Wolansky, and M. Mori. 2006. Patterns of transorbital intracranial injury: a review and comparison of occult and non-occult cases. Survey of Ophthalmology 51:449-460.

7. Levy M. L. 2000. Outcome prediction following penetrating craniocerebral injury in a civilian population: aggressive surgical management in patients with admission Glasgow Coma Scale scores of 6 to 15. Neurosurgical Focus 8:1-6.

8. Loggini A., V. I. Vasenina, A. Mansour, P. Das, P. M. Horowitz, F. D. Goldenberg, et al. 2020. Management of civilians with penetrating brain injury: A systematic review. Journal of Critical Care 56:159-166.

\section{Figure Legends:}

Figure 1: Frontal views (preoperative and 6 months postoperative)

(left) Preoperative and (right) 6 months post-surgery, frontal view. Note that the white arrow was an insertion point.

Figure 2: Computed tomography sagittal views (preoperative and 6 months postoperative)

Sagittal views of a computed tomography scan, preoperative (left) and 6 months after surgery (right). Note that the skull base defect was reconstructed.
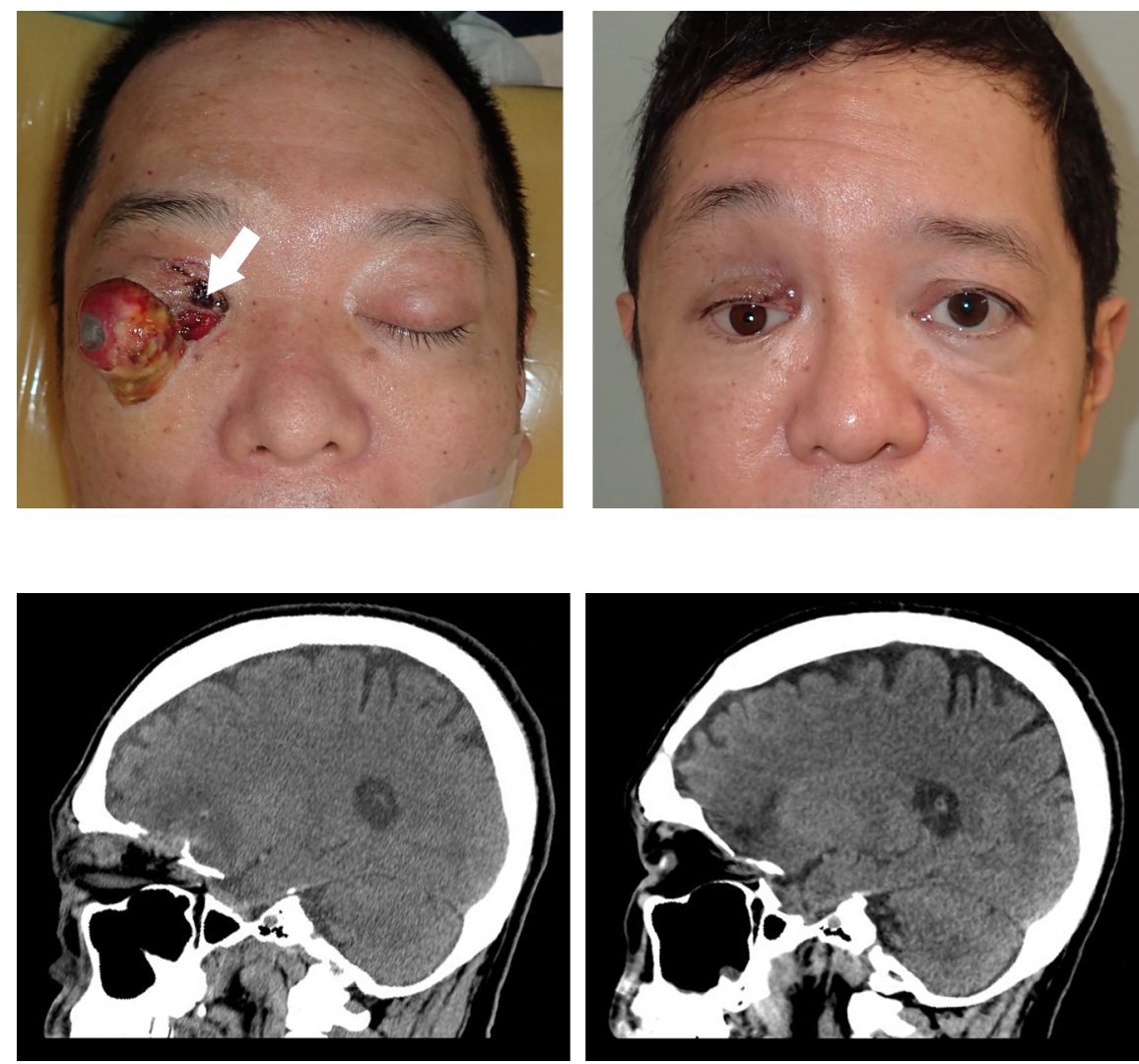